\title{
A GENERALIZATION OF THE CONCEPT OF DISTANCE BASED ON THE SIMPLEX INEQUALITY
}

\author{
GERGELY KISS, JEAN-LUC MARICHAL, AND BRUNO TEHEUX
}

\begin{abstract}
We introduce and discuss the concept of $n$-distance, a generalization to $n$ elements of the classical notion of distance obtained by replacing the triangle inequality with the so-called simplex inequality

$$
d\left(x_{1}, \ldots, x_{n}\right) \leq K \sum_{i=1}^{n} d\left(x_{1}, \ldots, x_{n}\right)_{i}^{z}, \quad x_{1}, \ldots, x_{n}, z \in X,
$$

where $K=1$. Here $d\left(x_{1}, \ldots, x_{n}\right)_{i}^{z}$ is obtained from the function $d\left(x_{1}, \ldots, x_{n}\right)$ by setting its $i$ th variable to $z$. We provide several examples of $n$-distances, and for each of them we investigate the infimum of the set of real numbers $K \in] 0,1]$ for which the inequality above holds. We also introduce a generalization of the concept of $n$-distance obtained by replacing in the simplex inequality the sum function with an arbitrary symmetric function.
\end{abstract}

\section{INTRODUCTION}

The notion of metric space, as first introduced by Fréchet [13] and later developed by Hausdorff [14], is one of the key ingredients in many areas of pure and applied mathematics, particularly in analysis, topology, geometry, statistics, and data analysis.

Denote the half-line $\left[0,+\infty\left[\right.\right.$ by $\mathbb{R}_{+}$. Recall that a metric space is a pair $(X, d)$, where $X$ is a nonempty set and $d$ is a distance on $X$, that is, a function $d: X^{2} \rightarrow \mathbb{R}_{+}$satisfying the following conditions:

- $d\left(x_{1}, x_{2}\right) \leq d\left(x_{1}, z\right)+d\left(z, x_{2}\right)$ for all $x_{1}, x_{2}, z \in X$ (triangle inequality),

- $d\left(x_{1}, x_{2}\right)=d\left(x_{2}, x_{1}\right)$ for all $x_{1}, x_{2} \in X$ (symmetry),

- $d\left(x_{1}, x_{2}\right)=0$ if and only if $x_{1}=x_{2}$ (identity of indiscernibles).

Generalizations of the concept of distance in which $n \geq 3$ elements are considered have been investigated by several authors (see, e.g., [5, Chapter 3] and the references therein). The three conditions above may be generalized to $n$-variable functions $d: X^{n} \rightarrow \mathbb{R}_{+}$in the following ways. For any integer $n \geq 1$, we set $[n]=\{1, \ldots, n\}$. For any $i \in[n]$ and any $z \in X$, we denote by $d\left(x_{1}, \ldots, x_{n}\right)_{i}^{z}$ the function obtained from $d\left(x_{1}, \ldots, x_{n}\right)$ by setting its $i$ th variable to $z$. Let also denote by $S_{n}$ the set of all permutations on $[n]$. A function $d: X^{n} \rightarrow \mathbb{R}_{+}$is said to be an $(n-1)$-semimetric $[7]$ if it satisfies

(i) $d\left(x_{1}, \ldots, x_{n}\right) \leq \sum_{i=1}^{n} d\left(x_{1}, \ldots, x_{n}\right)_{i}^{z}$ for all $x_{1}, \ldots, x_{n}, z \in X$,

(ii) $d\left(x_{1}, \ldots, x_{n}\right)=d\left(x_{\pi(1)}, \ldots, x_{\pi(n)}\right)$ for all $x_{1}, \ldots, x_{n} \in X$ and all $\pi \in S_{n}$, and it is said to be an $(n-1)$-hemimetric [5,6] if additionally it satisfies

(iii') $d\left(x_{1}, \ldots, x_{n}\right)=0$ if and only if $x_{1}, \ldots, x_{n}$ are not pairwise distinct.

Date: January 11, 2018.

2010 Mathematics Subject Classification. Primary 39B72; Secondary 26D99.

Key words and phrases. $n$-distance, simplex inequality, Fermat point, smallest enclosing sphere.

Corresponding author: Bruno Teheux is with the Mathematics Research Unit, University of Luxembourg, Maison du Nombre, 6, avenue de la Fonte, L-4364 Esch-sur-Alzette, Luxembourg. Email: bruno.teheux[at]uni.lu. 
Condition (i) is referred to as the simplex inequality [5,7]. For $n=3$, this inequality can be interpreted as follows: the area of a triangle face of a tetrahedron does not exceed the sum of the areas of the remaining three faces.

The following variant of condition (iii') can also be naturally considered:

(iii) $d\left(x_{1}, \ldots, x_{n}\right)=0$ if and only if $x_{1}=\cdots=x_{n}$.

For $n=3$, functions satisfying conditions (i), (ii), and (iii) were introduced by Dhage [8] and called $D$-distances. Their topological properties were investigated subsequently [9[1], but unfortunately most of the claimed results are incorrect, see [23]. Moreover, it turned out that a stronger version of $D$-distance is needed for a sound topological use of these functions [16, 23, 24].

In this paper we introduce and discuss the following simultaneous generalization of the concepts of distance and $D$-distance by considering functions with $n \geq 2$ arguments.

Definition 1.1 (see [17]). Let $n \geq 2$ be an integer. We say that $(X, d)$ is an $n$-metric space if $X$ is a nonempty set and $d$ is an $n$-distance on $X$, that is, a function $d: X^{n} \rightarrow \mathbb{R}_{+}$ satisfying conditions (i), (ii), and (iii).

We observe that for any $n$-distance $d: X^{n} \rightarrow \mathbb{R}_{+}$, the set of real numbers $\left.\left.K \in\right] 0,1\right]$ for which the condition

$$
d\left(x_{1}, \ldots, x_{n}\right) \leq K \sum_{i=1}^{n} d\left(x_{1}, \ldots, x_{n}\right)_{i}^{z}, \quad x_{1}, \ldots, x_{n}, z \in X,
$$

holds has an infimum $K^{*}$. We call it the best constant associated with the $n$-distance $d$. Determining the value of $K^{*}$ for a given $n$-distance is an interesting problem that might be mathematically challenging. It is the purpose of this paper to provide natural examples of $n$-distances and to show how elegant the investigation of the values of the best constants might be.

It is worth noting that determining the best constant $K^{*}$ is not relevant for nonconstant $(n-1)$-hemimetrics because we always have $K^{*}=1$ for those functions. Indeed, we have

$$
0<d\left(x_{1}, \ldots, x_{n}\right)=\sum_{i=1}^{n} d\left(x_{1}, \ldots, x_{n}\right)_{i}^{x_{n}}
$$

for any pairwise distinct elements $x_{1}, \ldots, x_{n}$ of $X$.

The paper is organized as follows. In Section 2 we provide some basic properties of $n$-metric spaces as well as some examples of $n$-distances together with their corresponding best constants. In Section 3 we investigate the values of the best constants for Fermat point based $n$-distances and discuss the particular case of median graphs. In Section 4 we consider some geometric constructions (smallest enclosing sphere and number of directions) to define $n$-distances and study their corresponding best constants. In Section 5 we introduce a generalization of the concept of $n$-distance by replacing in condition (i) the sum function with an arbitrary symmetric $n$-variable function. Finally, in Section 6 we conclude the paper by proposing topics for further research.

Remark 1. A multidistance on $X$, as introduced by Martín and Mayor [19], is a function $d: \cup_{n \geqslant 1} X^{n} \rightarrow \mathbb{R}_{+}$such that, for every integer $n \geq 1$, the restriction of $d$ to $X^{n}$ satisfies conditions (ii), (iii), and

(i') $d\left(x_{1}, \ldots, x_{n}\right) \leqslant \sum_{i=1}^{n} d\left(x_{i}, z\right)$ for all $x_{1}, \ldots, x_{n}, z \in X$.

Properties of multidistances as well as instances including the Fermat multidistance and smallest enclosing ball multidistances have been investigated for example in [2, 18- -20]. Note that multidistances have an indefinite number of arguments whereas $n$-distances have 
a fixed number of arguments. In particular, an $n$-distance can be defined without referring to any given 2-distance. Interestingly, some of the $n$-distances we present in this paper cannot be constructed from the concept of multidistance (see Section 6).

\section{BASIC EXAMPLES AND GENERAL PROPERTIES OF $n$-DISTANCES}

Let us illustrate the concept of $n$-distance by giving a few elementary examples. Other classes of $n$-distances will be investigated in the next sections. We denote by $|E|$ the cardinalily of any set $E$.

Example 2.1 (Drastic $n$-distance). For every integer $n \geqslant 2$, the map $d: X^{n} \rightarrow \mathbb{R}_{+}$defined by $d\left(x_{1}, \ldots, x_{n}\right)=0$, if $x_{1}=\cdots=x_{n}$, and $d\left(x_{1}, \ldots, x_{n}\right)=1$, otherwise, is an $n$-distance on $X$ for which the best constant is $K_{n}^{*}=\frac{1}{n-1}$. Indeed, let $x_{1}, \ldots, x_{n}, z \in X$ and assume that $d\left(x_{1}, \ldots, x_{n}\right)=1$. If there exists $k \in[n]$ such that $x_{i}=x_{j} \neq x_{k}$ for all $i, j \in[n] \backslash\{k\}$, then we have

$$
\sum_{i=1}^{n} d\left(x_{1}, \ldots, x_{n}\right)_{i}^{z}= \begin{cases}n-1, & \text { if } z \in\left\{x_{1}, \ldots, x_{n}\right\} \backslash\left\{x_{k}\right\}, \\ n, & \text { otherwise. }\end{cases}
$$

In all other cases we have $\sum_{i=1}^{n} d\left(x_{1}, \ldots, x_{n}\right)_{i}^{z}=n$.

Example 2.2 (Cardinality based $n$-distance). For every integer $n \geqslant 2$, the map $d: X^{n} \rightarrow \mathbb{R}_{+}$ defined by

$$
d\left(x_{1}, \ldots, x_{n}\right)=\left|\left\{x_{1}, \ldots, x_{n}\right\}\right|-1
$$

is an $n$-distance on $X$ for which the best constant is $K_{n}^{*}=\frac{1}{n-1}$. Indeed, let $x_{1}, \ldots, x_{n}, z \epsilon$ $X$ and assume that $d\left(x_{1}, \ldots, x_{n}\right) \geq 1$. The case $n=2$ is trivial. So let us further assume that $n \geq 3$. For every $i \in[n]$, set $m_{i}=\left|\left\{j \in[n] \mid x_{j}=x_{i}\right\}\right|$. If $\left|\left\{x_{1}, \ldots, x_{n}\right\}\right|<n$ (which means that there exists $j \in[n]$ such that $m_{j} \geq 2$ ), then it is straightforward to see that

$$
\begin{aligned}
\sum_{i=1}^{n} d\left(x_{1}, \ldots, x_{n}\right)_{i}^{z} & \geq n d\left(x_{1}, \ldots, x_{n}\right)-\left|\left\{i \in[n] \mid m_{i}=1\right\}\right| \\
& \geq(n-1) d\left(x_{1}, \ldots, x_{n}\right),
\end{aligned}
$$

where the first inequality is an equality if and only if $z=x_{j}$ for some $j \in[n]$ such that $m_{j} \geq 2$, and the second inequality is an equality if and only if there is exactly one $j \in[n]$ such that $m_{j} \geq 2$. If $\left|\left\{x_{1}, \ldots, x_{n}\right\}\right|=n$, then

$$
\sum_{i=1}^{n} d\left(x_{1}, \ldots, x_{n}\right)_{i}^{z} \geq(n-1) d\left(x_{1}, \ldots, x_{n}\right),
$$

with equality if and only if $z \in\left\{x_{1}, \ldots, x_{n}\right\}$.

Example 2.3 (Diameter). Given a metric space $(X, d)$ and an integer $n \geqslant 2$, the map $d_{\text {max }}: X^{n} \rightarrow \mathbb{R}_{+}$defined by

$$
d_{\max }\left(x_{1}, \ldots, x_{n}\right)=\max _{\{i, j\} \subseteq[n]} d\left(x_{i}, x_{j}\right)
$$

is an $n$-distance on $X$ for which we have $K_{n}^{*}=\frac{1}{n-1}$. Indeed, let $x_{1}, \ldots, x_{n}, z \in X$ and assume without loss of generality that $d_{\max }\left(x_{1}, \ldots, x_{n}\right)=d\left(x_{1}, x_{2}\right)$. For every $i \in[n]$ we have

$$
d_{\max }\left(x_{1}, \ldots, x_{n}\right)_{i}^{z} \geq \begin{cases}d\left(x_{2}, z\right), & \text { if } i=1 \\ d\left(x_{1}, z\right), & \text { if } i=2, \\ d\left(x_{1}, x_{2}\right), & \text { otherwise. }\end{cases}
$$


Using the triangle inequality, we then obtain

$$
\begin{aligned}
\sum_{i=1}^{n} d_{\max }\left(x_{1}, \ldots, x_{n}\right)_{i}^{z} & \geq(n-2) d\left(x_{1}, x_{2}\right)+d\left(x_{1}, z\right)+d\left(x_{2}, z\right) \\
& \geq(n-1) d\left(x_{1}, x_{2}\right)=(n-1) d_{\max }\left(x_{1}, \ldots, x_{n}\right),
\end{aligned}
$$

which proves that $K_{n}^{*} \leq \frac{1}{n-1}$. To prove that $K_{n}^{*}=\frac{1}{n-1}$, note that if $x_{1}=\cdots=x_{n-1}=z$ and $x_{n} \neq z$, then $\sum_{i=1}^{n} d_{\max }\left(x_{1}, \ldots, x_{n}\right)_{i}^{z}=(n-1) d_{\max }\left(x_{1}, \ldots, x_{n}\right)$.

Example 2.4 (Sum based $n$-distance). Given a metric space $(X, d)$ and an integer $n \geq 2$, the map $d_{\Sigma}: X^{n} \rightarrow \mathbb{R}_{+}$defined by

$$
d_{\Sigma}\left(x_{1}, \ldots, x_{n}\right)=\sum_{\{i, j\} \subseteq[n]} d\left(x_{i}, x_{j}\right)
$$

is an $n$-distance on $X$ for which we have $K_{n}^{*}=\frac{1}{n-1}$. Indeed, for fixed $x_{1}, \ldots, x_{n}, z \in X$, we have

$$
\sum_{i=1}^{n} d_{\Sigma}\left(x_{1}, \ldots, x_{n}\right)_{i}^{z}=(n-2) \sum_{\{i, j\} \subseteq[n]} d\left(x_{i}, x_{j}\right)+(n-1) \sum_{i=1}^{n} d\left(x_{i}, z\right) .
$$

Using the triangle inequality we obtain

$$
(n-1) \sum_{i=1}^{n} d\left(x_{i}, z\right)=\sum_{\{i, j\} \subseteq[n]}\left(d\left(x_{i}, z\right)+d\left(x_{j}, z\right)\right) \geq \sum_{\{i, j\} \subseteq[n]} d\left(x_{i}, x_{j}\right) .
$$

Therefore, we finally obtain

$$
\sum_{i=1}^{n} d_{\Sigma}\left(x_{1}, \ldots, x_{n}\right)_{i}^{z} \geq(n-1) \sum_{\{i, j\} \subseteq[n]} d\left(x_{i}, x_{j}\right)=(n-1) d_{\Sigma}\left(x_{1}, \ldots, x_{n}\right),
$$

which proves that $K_{n}^{*} \leq \frac{1}{n-1}$. To prove that $K_{n}^{*}=\frac{1}{n-1}$, note that if $x_{1}=\cdots=x_{n-1}=z$ and $x_{n} \neq z$, then $\sum_{i=1}^{n} d_{\Sigma}\left(x_{1}, \ldots, x_{n}\right)_{i}^{z}=(n-1) d_{\Sigma}\left(x_{1}, \ldots, x_{n}\right)$.

Example 2.5 (Arithmetic mean based $n$-distance). For any integer $n \geq 2$, the map $d: \mathbb{R}^{n} \rightarrow$ $\mathbb{R}_{+}$defined by

$$
d\left(x_{1}, \ldots, x_{n}\right)=\frac{1}{n} \sum_{i=1}^{n} x_{i}-x_{(1)}=\frac{1}{n} \sum_{i=1}^{n}\left(x_{i}-x_{(1)}\right),
$$

where $x_{(1)}=\min \left\{x_{1}, \ldots, x_{n}\right\}$, is an $n$-distance on $\mathbb{R}$ for which $K_{n}^{*}=\frac{1}{n-1}$. Indeed, let $x_{1}, \ldots, x_{n}, z \in \mathbb{R}$. By symmetry of $d$ we may assume that $x_{1} \leq \cdots \leq x_{n}$. We then obtain

$$
d\left(x_{1}, \ldots, x_{n}\right)=\frac{1}{n}\left(\sum_{i=1}^{n} x_{i}\right)-x_{1}
$$

and

$$
\sum_{i=1}^{n} d\left(x_{1}, \ldots, x_{n}\right)_{i}^{z}=\left(1-\frac{1}{n}\right)\left(\sum_{i=1}^{n} x_{i}\right)+z-(n-1) \min \left\{x_{1}, z\right\}-\min \left\{x_{2}, z\right\} .
$$

It follows that condition (1) holds for $K_{n}=\frac{1}{n-1}$ if and only if

$$
(n-1)\left(x_{1}-\min \left\{x_{1}, z\right\}\right)+\left(z-\min \left\{x_{2}, z\right\}\right) \geq 0 .
$$

We then observe that this inequality is trivially satisfied, which proves that $K_{n}^{*} \leq \frac{1}{n-1}$. To prove that $K_{n}^{*}=\frac{1}{n-1}$, just take $x_{1}, \ldots, x_{n}, z \in \mathbb{R}$ so that $x_{1}<z<x_{2}=\cdots=x_{n}$.

In the next result, we show how to construct an $(n-1)$-hemimetric from an $n$-distance. 
Proposition 2.6. Let $(X, d)$ be an n-metric space for some integer $n \geq 2$. The function $d^{\prime}: X^{n} \rightarrow \mathbb{R}_{+}$defined as

$$
d^{\prime}\left(x_{1}, \ldots, x_{n}\right)= \begin{cases}0, & \text { if } x_{1}, \ldots, x_{n} \text { are not pairwise distinct, } \\ d\left(x_{1}, \ldots, x_{n}\right), & \text { otherwise, }\end{cases}
$$

is an (n-1)-hemimetric.

Proof. It is easy to see that $d^{\prime}$ satisfies conditions (ii) and (iii'). To see that condition (i) holds, let $x_{1}, \ldots, x_{n}, z \in X$ and assume that $d^{\prime}\left(x_{1}, \ldots, x_{n}\right)>0$. If $d^{\prime}\left(x_{1}, \ldots, x_{n}\right)_{i}^{z}=$ $d\left(x_{1}, \ldots, x_{n}\right)_{i}^{z}$ for every $i \in[n]$, then the simplex inequality holds for $d^{\prime}$. Otherwise, we must have $z \in\left\{x_{1}, \ldots, x_{n}\right\}$ and then $\sum_{i=1}^{n} d^{\prime}\left(x_{1}, \ldots, x_{n}\right)_{i}^{z}=d^{\prime}\left(x_{1}, \ldots, x_{n}\right)$. This shows that condition (i) holds.

The next proposition shows that two of the standard constructions of distances from existing ones are still valid for $n$-distances. The proof uses the following lemma.

Lemma 2.7. For any $a_{1}, \ldots, a_{n}, a \in \mathbb{R}_{+}$such that $a \leq \sum_{i=1}^{n} a_{i}$, we have

$$
\frac{a}{1+a} \leq \sum_{i=1}^{n} \frac{a_{i}}{1+a_{i}}
$$

Proof. We proceed by induction on $n \geq 1$. The result is easily obtained for $n \in\{1,2\}$. Assume that the result holds for $k \in\{1, \ldots, n-1\}$ for some $n \geq 3$, and that $a \leq \sum_{i=1}^{n} a_{i}$ for some $a, a_{1}, \ldots, a_{n} \in \mathbb{R}_{+}$. Letting $b=\max \left\{0, a-a_{n}\right\}$, we obtain

$$
\frac{a}{1+a} \leq \frac{b}{1+b}+\frac{a_{n}}{1+a_{n}} \leq \sum_{i=1}^{n} \frac{a_{i}}{1+a_{i}}
$$

where the first inequality is obtained by the induction hypothesis applied to $a \leq b+a_{n}$, and the second to $b \leq \sum_{i=1}^{n-1} a_{i}$.

Proposition 2.8. Let $d$ and $d^{\prime}$ be $n$-distances on $X$ and let $\lambda>0$. The following assertions hold.

(a) $d+d^{\prime}$ and $\lambda d$ are $n$-distances on $X$.

(b) $\frac{d}{1+d}$ is an $n$-distance on $X$, with values in $[0,1]$.

Proof. (a) is a simple verification. For (b) we note that condition (i) holds for $\frac{d}{1+d}$ by Lemma 2.7

Remark 2. In the same spirit as Proposition 2.8 we observe that if $d: X \rightarrow \mathbb{R}_{+}$is an $n$ distance and $d_{0}: X \rightarrow \mathbb{R}_{+}$is an $(n-1)$-hemimetric, then $d+d_{0}$ is an $n$-distance.

\section{FERMAT POINT BASED $n$-DISTANCES}

Recall that, given a metric space $(X, d)$ and an integer $n \geq 2$, the Fermat set $F_{Y}$ of any $n$-element subset $Y=\left\{x_{1}, \ldots, x_{n}\right\}$ of $X$ is defined as

$$
F_{Y}=\left\{x \in X \mid \sum_{i=1}^{n} d\left(x_{i}, x\right) \leq \sum_{i=1}^{n} d\left(x_{i}, z\right) \text { for all } z \in X\right\} .
$$

Elements of $F_{Y}$ are the Fermat points of $Y$. The problem of finding the Fermat point of a triangle in the Euclidean plane was formulated by Fermat in the early 17th century, and was first solved by Torricelli around 1640. The general problem stated for $n \geq 2$ in any metric space was considered by many authors, and applications were found for instance in geometry, combinatorial optimization, and facility location. We refer to [3, Chapter II] 
and [12] for an account of the history of this problem. Also, in [15], the location problem is extended in various directions and studied also for very general metrics - more general than those of normed spaces.

We observe that $F_{Y}$ need not be nonempty in a general metric space. However, it follows from the continuity of the function $h: X \rightarrow \mathbb{R}_{+}$defined by $h(x)=\sum_{i=1}^{n} d\left(x_{i}, x\right)$ that $F_{Y}$ is nonempty whenever $(X, d)$ is a proper metric space. (Recall that a metric space is proper if every closed ball is compact.) In this section we will therefore assume that $(X, d)$ is a proper metric space.

Proposition 3.1. For any proper metric space $(X, d)$ and any integer $n \geq 2$, the map $d_{F}: X^{n} \rightarrow \mathbb{R}_{+}$defined as

$$
d_{F}\left(x_{1}, \ldots, x_{n}\right)=\min _{x \in X} \sum_{i=1}^{n} d\left(x_{i}, x\right),
$$

is an $n$-distance on $X$ and we call it the Fermat $n$-distance.

Proof. The map $d_{F}$ clearly satisfies conditions (ii) and (iii). Let us show that it satisfies condition (i). Assume first that $n=2$ and let $y_{1}, y_{2} \in X$ be such that

$$
d_{F}\left(z, x_{2}\right)=d\left(z, y_{1}\right)+d\left(x_{2}, y_{1}\right) \text { and } d_{F}\left(x_{1}, z\right)=d\left(x_{1}, y_{2}\right)+d\left(z, y_{2}\right) \text {. }
$$

By applying the triangle inequality, we obtain

$$
\begin{aligned}
d_{F}\left(z, x_{2}\right)+d_{F}\left(x_{1}, z\right) & =\left(d\left(x_{1}, y_{2}\right)+d\left(z, y_{2}\right)\right)+\left(d\left(z, y_{1}\right)+d\left(x_{2}, y_{1}\right)\right) \\
& \geq d\left(x_{1}, x_{2}\right)=d\left(x_{1}, x_{1}\right)+d\left(x_{1}, x_{2}\right) \geq d_{F}\left(x_{1}, x_{2}\right) .
\end{aligned}
$$

Assume now that $n \geq 3$ and let $y_{1}, \ldots, y_{n} \in X$ be such that

$$
d_{F}\left(x_{1}, \ldots, x_{n}\right)_{i}^{z}=\sum_{j \neq i} d\left(x_{j}, y_{i}\right)+d\left(z, y_{i}\right), \quad i=1, \ldots, n .
$$

It follows that

$$
\begin{aligned}
\sum_{i=1}^{n} d_{F}\left(x_{1}, \ldots, x_{n}\right)_{i}^{z} & \geq \sum_{i=1}^{n} \sum_{j \neq i} d\left(x_{j}, y_{i}\right) \\
& \geq\left(d\left(x_{1}, y_{n}\right)+d\left(x_{2}, y_{n}\right)\right)+\sum_{i=2}^{n-1}\left(d\left(x_{1}, y_{i}\right)+d\left(x_{i+1}, y_{i}\right)\right),
\end{aligned}
$$

that is, by applying the triangle inequality,

$$
\sum_{i=1}^{n} d_{F}\left(x_{1}, \ldots, x_{n}\right)_{i}^{z} \geq \sum_{i=2}^{n} d\left(x_{1}, x_{i}\right)=\sum_{i=1}^{n} d\left(x_{1}, x_{i}\right) \geq d_{F}\left(x_{1}, \ldots, x_{n}\right),
$$

where the last inequality follows from the definition of $d_{F}$.

In the next proposition we use rough counting arguments to obtain bounds for the best constant $K_{n}^{*}$ associated with the Fermat $n$-distance.

Proposition 3.2. For every $n \geq 2$, the best constant $K_{n}^{*}$ associated with the Fermat $n$ distance satisfies the inequalities $\frac{1}{n-1} \leq K_{n}^{*} \leq \frac{1}{[n / 2]}$.

Proof. Let $x_{1}, \ldots, x_{n} \in X$ and let $z$ be a Fermat point of $\left\{x_{1}, \ldots, x_{n}\right\}$. For every $i \in[n]$, denote by $y_{i}$ a Fermat point of $\{z\} \cup\left\{x_{1}, \ldots, x_{n}\right\} \backslash\left\{x_{i}\right\}$. We then have

$$
\begin{aligned}
d_{F}\left(x_{1}, \ldots, x_{n}\right)_{i}^{z} & =\sum_{j \neq i} d\left(x_{j}, y_{i}\right)+d\left(z, y_{i}\right) \\
& \leq \sum_{j \neq i} d\left(x_{j}, z\right)+d(z, z)=\sum_{j \neq i} d\left(x_{j}, z\right) .
\end{aligned}
$$


By summing over $i=1, \ldots, n$, we obtain

$$
\sum_{i=1}^{n} d_{F}\left(x_{1}, \ldots, x_{n}\right)_{i}^{z} \leq(n-1) \sum_{i=1}^{n} d\left(x_{i}, z\right)=(n-1) d_{F}\left(x_{1}, \ldots, x_{n}\right),
$$

which shows that $K_{n}^{*} \geq 1 /(n-1)$.

Now, if $z$ denotes any element of $X$ and if $y_{1}, \ldots, y_{n}$ are defined as in the first part of the proof, the identity (2) holds for every $i \in[n]$. Then, for $i=1, \ldots, n-1$, we have

$d_{F}\left(x_{1}, \ldots, x_{n}\right)_{i}^{z}+d_{F}\left(x_{1}, \ldots, x_{n}\right)_{i+1}^{z} \geq d\left(z, y_{i}\right)+d\left(z, y_{i+1}\right)+d\left(x_{i}, y_{i+1}\right)+\sum_{j \neq i} d\left(x_{j}, y_{i}\right)$

$$
\begin{aligned}
& \geq d\left(x_{i}, y_{i}\right)+\sum_{j \neq i} d\left(x_{j}, y_{i}\right) \\
& \geq d_{F}\left(x_{1}, \ldots, x_{n}\right),
\end{aligned}
$$

where (3) is obtained by a double application of the triangle inequality and (4) is obtained by definition of $d_{F}$.

It follows from (4) that $\sum_{i=1}^{n} d_{F}\left(x_{1}, \ldots, x_{n}\right)_{i}^{z} \geq\lfloor n / 2\rfloor d_{F}\left(x_{1}, \ldots, x_{n}\right)$, which proves that $K_{n}^{*} \leq\lfloor n / 2\rfloor^{-1}$.

The next proposition uses a more refined counting argument to provide an improvement of the upper bound obtained for $K_{n}^{*}$ in Proposition 3.2 Let us first state an immediate generalization of the hand-shaking lemma, which is folklore in graph theory.

Lemma 3.3. Let $G=(V, E, w)$ be a weighted simple graph, where $w: E \rightarrow \mathbb{R}_{+}$is the weighting function. If $f: V \rightarrow \mathbb{R}_{+}$is such that $f(x)+f(y) \geq w(e)$ for every $e=\{x, y\} \in E$, then

$$
\sum_{x \in V} f(x) \operatorname{deg}_{G}(x) \geq \sum_{e \in E} w(e),
$$

where $\operatorname{deg}_{G}(x)$ is the degree of $x$ in $G$.

Proposition 3.4. For every $n \geq 2$, the best constant $K_{n}^{*}$ associated with the Fermat $n$ distance satisfies $K_{n}^{*} \leq(4 n-4) /\left(3 n^{2}-4 n\right)$.

Proof. Let $z, x_{1}, \ldots, x_{n}, y, y_{1}, \ldots, y_{n} \in X$ be such that $y$ is a Fermat point of $\left\{x_{1}, \ldots, x_{n}\right\}$ and such that equation (2) holds for every $i \in[n]$. For any distinct $i, j \in[n]$, by the triangle inequality we have

$$
d\left(z, y_{i}\right)+d\left(z, y_{j}\right)+d\left(x_{i}, y_{j}\right) \geq d\left(x_{i}, y_{i}\right) .
$$

By summing (5) over all $j \in[n] \backslash\{i\}$ we obtain

$$
(n-1) d\left(z, y_{i}\right)+\sum_{j \neq i}\left(d\left(z, y_{j}\right)+d\left(x_{i}, y_{j}\right)\right) \geq(n-1) d\left(x_{i}, y_{i}\right) .
$$

By summing [6) over all $i \in[n]$ we then obtain

$$
2(n-1) \sum_{i=1}^{n} d\left(z, y_{i}\right)+\sum_{i=1}^{n} \sum_{j \neq i} d\left(x_{j}, y_{i}\right) \geq(n-1) \sum_{i=1}^{n} d\left(x_{i}, y_{i}\right) .
$$


Let us set $S=\sum_{i=1}^{n} \sum_{j \neq i} d\left(x_{j}, y_{i}\right)$. We then have

$$
\begin{aligned}
2(n-1) \sum_{i=1}^{n} d_{F}\left(x_{1}, \ldots, x_{n}\right)_{i}^{z} & =(2 n-3) S+S+2(n-1) \sum_{i=1}^{n} d\left(z, y_{i}\right) \\
& \geq(2 n-3) S+(n-1) \sum_{i=1}^{n} d\left(x_{i}, y_{i}\right) \\
& =(n-2) S+(n-1) \sum_{i=1}^{n} \sum_{j=1}^{n} d\left(x_{j}, y_{i}\right),
\end{aligned}
$$

where (8) follows by the definitions of $S$ and $d_{F}$, (9) follows by (7), and (10) by the definition of $S$.

Now, on the one hand, by the definition of $d_{F}$ we have

$$
(n-1) \sum_{i=1}^{n} \sum_{j=1}^{n} d\left(x_{j}, y_{i}\right) \geq n(n-1) d_{F}\left(x_{1}, \ldots, x_{n}\right) .
$$

On the other hand, let us fix $i \in[n]$ and set $V=\left\{x_{1}, \ldots, x_{n}\right\} \backslash\left\{x_{i}\right\}$. Define the function $f: V \rightarrow \mathbb{R}_{+}$by $f\left(x_{j}\right)=d\left(x_{j}, y_{i}\right)$ for any $j \neq i$, and consider the complete weighted graph $G=\left(V,\left(\begin{array}{c}V \\ 2\end{array}\right), w\right)$ defined by $w\left(\left\{x_{\ell}, x_{j}\right\}\right)=d\left(x_{\ell}, x_{j}\right)$ for any distinct $x_{\ell}, x_{k} \in V$. It follows from Lemma 3.3 that

$$
(n-2) \sum_{j \neq i} d\left(x_{j}, y_{i}\right) \geq \sum_{\left\{x_{k}, x_{\ell}\right\} \in\left(\begin{array}{c}
V \\
2
\end{array}\right)} d\left(x_{k}, x_{\ell}\right) .
$$

By summing (12) over all $i \in[n]$, we get

$$
\begin{aligned}
(n-2) S & \geq(n-2) \sum_{\{k, \ell\} \in\left(\begin{array}{c}
n \\
2
\end{array}\right)} d\left(x_{k}, x_{\ell}\right)=\frac{n-2}{2} \sum_{k=1}^{n} \sum_{\ell=1}^{n} d\left(x_{k}, x_{\ell}\right) \\
& \geq n \frac{n-2}{2} d_{F}\left(x_{1}, \ldots, x_{n}\right),
\end{aligned}
$$

where (13) is obtained by definition of $d_{F}$. By substituting (11) and (13) into (10), we finally obtain

$$
\sum_{i=1}^{n} d_{F}\left(x_{1}, \ldots, x_{n}\right)_{i}^{z} \geq \frac{n(3 n-4)}{4(n-1)} d_{F}\left(x_{1}, \ldots, x_{n}\right),
$$

which proves that $K_{n}^{*} \leq(4 n-4) /\left(3 n^{2}-4 n\right)$.

We observe that Proposition 3.4 provides a better upper bound than Proposition 3.2 for every $n \geq 2$, but the difference between these bounds converges to zero as $n$ tends to infinity. The high number of inequalities involved in the proof of Proposition 3.4 suggests that it is in general very difficult to obtain the exact value of $K_{n}^{*}$ (we have to find $x_{1}, \ldots, x_{n}, z \in X$ that turn these inequalities into equalities). However, we will now show that we can determine the value of $K_{n}^{*}$ when $d_{F}$ is the Fermat $n$-distance associated with the distance function in median graphs.

Recall that a median graph is a connected undirected simple graph in which, for any triplet of vertices $u, v, w$, there is one and only one vertex $\mathbf{m}(u, v, w)$ that is at the intersection of shortest paths between any two elements among $u, v, w$. Cubes and trees are instances of median graphs. In a median graph $G=(V, E)$, the Fermat 3-distance is the function $d_{\mathbf{m}}: V^{3} \rightarrow \mathbb{R}_{+}$defined by

$$
d_{\mathbf{m}}(u, v, w)=\min _{y \in V}(d(u, y)+d(v, y)+d(w, y)),
$$

where $d$ denotes the usual distance function between vertices in a connected graph. 
Proposition 3.5. If $G=(V, E)$ is a median graph, then the best constant $K^{*}$ associated with its Fermat 3-distance $d_{\mathbf{m}}$ is equal to $\frac{1}{2}$. Moreover, the only Fermat point of $\{u, v, w\}$ is $\mathbf{m}(u, v, w)$.

Proof. The minimum in (14) is realized by any $y_{0} \in V$ that realizes the minimum of the values

$$
(d(u, y)+d(v, y))+(d(w, y)+d(u, y))+(d(v, y)+d(w, y))
$$

for $y \in V$. By definition, the vertex $y_{0}=\mathbf{m}(u, v, w)$ is on shortest paths between any two elements among $u, v, w$, which shows that it realizes the minimum of each of the three terms in (15), and hence the minimum in (14).

It follows that

$$
\begin{aligned}
d_{\mathbf{m}}(u, v, z) & =d\left(u, y_{0}\right)+d\left(v, y_{0}\right)+d\left(z, y_{0}\right) \\
& =\frac{1}{2}\left(d\left(u, y_{0}\right)+d\left(v, y_{0}\right)+d\left(z, y_{0}\right)+d\left(u, y_{0}\right)+d\left(v, y_{0}\right)+d\left(z, z_{0}\right)\right) \\
& =\frac{1}{2}(d(u, v)+d(u, z)+d(v, z)),
\end{aligned}
$$

which shows that $\min _{z \in V} d_{\mathbf{m}}(u, v, z)$ is equal to $d(u, v)$, and is realized by any element $z_{0}$ on a shortest path between $u$ and $v$. We conclude that the minimum of

$$
d_{\mathbf{m}}(z, v, w)+d_{\mathbf{m}}(u, z, w)+d_{\mathbf{m}}(u, v, z)
$$

for $z \in V$ is realized by $z_{0}=\mathbf{m}(u, v, w)$, and is equal to $d(v, w)+d(u, w)+d(u, v)=$ $2 d_{\mathbf{m}}(u, v, w)$. We have proved that the best constant $K^{*}$ associated with $d_{\mathbf{m}}$ is $\frac{1}{2}$.

\section{EXAMPLES OF $n$-DISTANCES BASED ON GEOMETRIC CONSTRUCTIONS}

In this section we introduce $n$-distances defined from certain geometric constructions and investigate their corresponding best constants. In what follows, we denote by $d$ the Euclidean distance on $\mathbb{R}^{k}$ for some integer $k \geq 2$.

The first $n$-distances we investigate are based on the following construction.

Definition 4.1. For any $n \geq 2$ and any $x_{1}, \ldots, x_{n} \in \mathbb{R}^{k}$, we denote by $S\left(x_{1}, \ldots, x_{n}\right)$ the smallest $(k-1)$-dimensional sphere enclosing $\left\{x_{1}, \ldots, x_{n}\right\}$. For any $i \in[n]$ and any $z \in \mathbb{R}^{k}$, we denote by $S\left(x_{1}, \ldots, x_{n}\right)_{i}^{z}$ the smallest $(k-1)$-dimensional sphere enclosing $\left\{x_{1}, \ldots, x_{i-1}, z, x_{i+1}, \ldots, x_{n}\right\}$.

The sphere introduced in Definition 4.1 always exists and is unique. Moreover, it can be computed in linear time [21,22] or expected linear time [26].

When $k=2$, we have the following fact.

Fact 4.2. Let $A, B, C$ be the vertices of a triangle in $\mathbb{R}^{2}$.

(a) If $A B C$ forms an acute triangle with angles $\alpha, \beta$ and $\gamma$, respectively, then $S(A, B, C)$ is the circumcircle $\mathcal{C}$ of $A B C$ whose radius $R$ satisfies

$$
R=\frac{a}{2 \sin \alpha}=\frac{b}{2 \sin \beta}=\frac{c}{2 \sin \gamma},
$$

where $a=d(B, C), b=d(A, C)$, and $c=d(A, B)$. Let $A^{*}$ be one of the two points of the circle $\mathcal{C}$ that is on the bisector of $B C$. Then the perimeter of the triangle $A B C$ strictly decreases as $A$ moves along $\mathcal{C}$ from $A^{*}$ to $B$ (or from $A^{*}$ to C). 
(b) If $A B C$ is obtuse in $A$, then $S(A, B, C)$ contains $B$ and $C$, and its diameter is equal to a.

(c) It follows from (a) and (b) that the radius $R$ of $S(A, B, C)$ satisfies

$$
R \geq \max \left\{\frac{a}{2}, \frac{b}{2}, \frac{c}{2}\right\} .
$$

Proposition 4.3 (Radius of $S\left(x_{1}, \ldots, x_{n}\right)$ in $\left.\mathbb{R}^{2}\right)$. For any $n \geq 2$, the map $d_{r}:\left(\mathbb{R}^{2}\right)^{n} \rightarrow \mathbb{R}_{+}$ that associates with any $\left(x_{1}, \ldots, x_{n}\right) \in\left(\mathbb{R}^{2}\right)^{n}$ the radius of $S\left(x_{1}, \ldots, x_{n}\right)$ is an $n$-distance for which we have $K_{n}^{*}=\frac{1}{n-1}$.

Proof. Let us show that the map $d_{r}$ satisfies the simplex inequality for $K_{n}=\frac{1}{n-1}$. Since $d_{r}$ is a continuous function, we can assume that its arguments are pairwise distinct.

Consider first the case where $n=2$. For any distinct $A, B \in \mathbb{R}^{2}$, we have $d_{r}(A, B)=$ $\frac{1}{2} d(A, B)$, which proves that the simplex inequality holds for $n=2$.

Suppose now that $n=3$ and let us show that, for any $A, B, C, Z \in \mathbb{R}^{2}$, with $A, B, C$ pairwise distinct, we have

$$
2 d_{r}(A, B, C) \leq d_{r}(Z, B, C)+d_{r}(A, Z, C)+d_{r}(A, B, Z) .
$$

Set $a=d(B, C), b=d(A, C)$, and $c=d(A, B)$. By 177 we have

$$
d_{r}(Z, B, C) \geq \frac{a}{2}, \quad d_{r}(A, Z, C) \geq \frac{b}{2}, \quad d_{r}(A, B, Z) \geq \frac{c}{2},
$$

and hence

$$
d_{r}(Z, B, C)+d_{r}(A, Z, C)+d_{r}(A, B, Z) \geq \frac{a+b+c}{2} \geq \max \{a, b, c\} .
$$

Suppose first that $A B C$ is not acute, assuming for instance that $\beta \geq \frac{\pi}{2}$. Then $2 d_{r}(A, B, C)=$ $b$, and then (18) immediately follows from (20). Suppose now that $A B C$ is acute, with circumcircle $\mathcal{C}$, and consider the triangle $A^{\prime} B C$, with sides $a, b^{\prime}, c^{\prime}$, such that $A^{\prime} \in \mathcal{C}$ and $\varangle A^{\prime} B C=\frac{\pi}{2}$. By Fact 4.2 (a) we have

$$
\frac{a+b+c}{2} \geq \frac{a+b^{\prime}+c^{\prime}}{2} \geq b^{\prime}=2 d_{r}\left(A^{\prime}, B, C\right)=2 d_{r}(A, B, C),
$$

and then again (18) follows from (20). Finally, the equality is obtained in (18) by taking $A \neq B=C=Z$.

We now prove the general case where $n \geq 3$. Let $A_{1}, \ldots, A_{n}, Z \in \mathbb{R}^{2}$, with $A_{1}, \ldots, A_{n}$ pairwise distinct. It is a known fact [4] that either there are $j, k \in[n]$ such that $A_{j}$ and $A_{k}$ are distinct and

$$
S\left(A_{1}, \ldots, A_{n}\right)=S\left(A_{j}, A_{k}\right)
$$

or there are $j, k, \ell \in[n]$ such that $A_{j}, A_{k}$, and $A_{\ell}$ are distinct and

$$
S\left(A_{1}, \ldots, A_{n}\right)=S\left(A_{j}, A_{k}, A_{\ell}\right) .
$$

Let us consider the latter case (the proof in the former case can be dealt with similarly). On the one hand, using (17) it is easy to see that

$$
d_{r}\left(A_{1}, \ldots, A_{n}\right)_{i}^{Z} \geq d_{r}\left(A_{1}, \ldots, A_{n}\right), \quad i \notin\{j, k, \ell\} .
$$

On the other hand, the following inequalities hold:

$$
\begin{aligned}
& d_{r}\left(A_{1}, \ldots, A_{n}\right)_{j}^{Z} \geq d_{r}\left(Z, A_{k}, A_{\ell}\right), \\
& d_{r}\left(A_{1}, \ldots, A_{n}\right)_{k}^{Z} \geq d_{r}\left(A_{j}, Z, A_{\ell}\right), \\
& d_{r}\left(A_{1}, \ldots, A_{n}\right)_{\ell}^{Z} \geq d_{r}\left(A_{j}, A_{k}, Z\right) .
\end{aligned}
$$


Indeed, $S\left(A_{1}, \ldots, A_{n}\right)_{j}^{Z}$ encloses the points $Z, A_{k}$, and $A_{\ell}$ and hence cannot have a radius strictly smaller than that of $S\left(Z, A_{k}, A_{\ell}\right)$.

Adding up these inequalities and then using (18), we obtain

$$
\begin{aligned}
& d_{r}\left(A_{1}, \ldots, A_{n}\right)_{j}^{Z}+d_{r}\left(A_{1}, \ldots, A_{n}\right)_{k}^{Z}+d_{r}\left(A_{1}, \ldots, A_{n}\right)_{\ell}^{Z} \\
& \quad \geq d_{r}\left(Z, A_{k}, A_{\ell}\right)+d_{r}\left(A_{j}, Z, A_{\ell}\right)+d_{r}\left(A_{j}, A_{k}, Z\right) \\
& \quad \geq 2 d_{r}\left(A_{j}, A_{k}, A_{\ell}\right)=2 d_{r}\left(A_{1}, \ldots, A_{n}\right) .
\end{aligned}
$$

Combining 21] with 221, we finally obtain

$$
\sum_{i=1}^{n} d_{r}\left(A_{1}, \ldots, A_{n}\right)_{i}^{Z} \geq(n-1) d_{r}\left(A_{1}, \ldots, A_{n}\right),
$$

which proves that $K_{n}^{*} \leq \frac{1}{n-1}$. To prove that $K_{n}^{*}=\frac{1}{n-1}$, just consider $A_{2}=\cdots=A_{n}=Z$ and $A_{1} \neq A_{2}$.

Proposition 4.4 (Area bounded by $S\left(x_{1}, \ldots, x_{n}\right)$ in $\mathbb{R}^{2}$ ). For any $n \geq 3$, the map $d_{s}:\left(\mathbb{R}^{2}\right)^{n} \rightarrow$ $\mathbb{R}_{+}$that associates with any $\left(x_{1}, \ldots, x_{n}\right) \in\left(\mathbb{R}^{2}\right)^{n}$ the surface area bounded by $S\left(x_{1}, \ldots, x_{n}\right)$ is an $n$-distance for which we have $K_{n}^{*}=\left(n-\frac{3}{2}\right)^{-1}$.

Proof. Let us show that the map $d_{s}=\pi d_{r}^{2}$ satisfies the simplex inequality with constant $K_{n}=\left(n-\frac{3}{2}\right)^{-1}$. Since $d_{r}$ is continuous, we can assume that its arguments are pairwise distinct.

Consider first the case where $n=3$ and let us show that, for any $A, B, C, Z \in \mathbb{R}^{2}$, with $A, B, C$ pairwise distinct, we have

$$
d_{r}(A, B, C)^{2} \leq \frac{2}{3}\left(d_{r}(Z, B, C)^{2}+d_{r}(A, Z, C)^{2}+d_{r}(A, B, Z)^{2}\right) .
$$

If the triangle $A B C$ is acute, then we may assume for instance that $\frac{\pi}{3} \leq \alpha \leq \frac{\pi}{2}$, which implies $\frac{\sqrt{3}}{2} \leq \sin \alpha \leq 1$. Using (16), we then have

$$
d_{r}(A, B, C)^{2} \leq \frac{a^{2}}{3} \leq \frac{2}{3}\left(\frac{a^{2}}{4}+\frac{a^{2}}{4}\right) \leq \frac{2}{3}\left(\frac{a^{2}}{4}+\frac{b^{2}}{4}+\frac{c^{2}}{4}\right),
$$

where the latter inequality holds by the law of cosines. We then obtain (23) by combining (19) with 24).

If $A B C$ is obtuse in $C$, then $d_{r}(A, B, C)=\frac{c}{2}$. Using the triangle inequality and the square and arithmetic mean inequality, we also have

$$
\frac{a^{2}+b^{2}}{2} \geq\left(\frac{a+b}{2}\right)^{2} \geq \frac{c^{2}}{4}
$$

Combining these observations with [19], we obtain

$$
\begin{aligned}
& \frac{2}{3}\left(d_{r}(Z, B, C)^{2}+d_{r}(A, Z, C)^{2}+d_{r}(A, B, Z)^{2}\right) \\
& \quad \geq \frac{2}{3}\left(\frac{a^{2}}{4}+\frac{b^{2}}{4}+\frac{c^{2}}{4}\right) \geq \frac{2}{3} \frac{3}{8} c^{2}=\left(\frac{c}{2}\right)^{2}=d_{r}(A, B, C)^{2} .
\end{aligned}
$$

To see that the general case where $n \geq 3$ also holds, it suffices to proceed as in the proof of Proposition 4.3. This shows that $K_{n}^{*} \leq\left(n-\frac{3}{2}\right)^{-1}$. To prove that $K_{n}^{*}=\left(n-\frac{3}{2}\right)^{-1}$, just consider $A_{1} \neq A_{2}$ and $A_{3}=\cdots=A_{n}=Z=\left(A_{1}+A_{2}\right) / 2$, where $\left(A_{1}+A_{2}\right) / 2$ is the midpoint of $A_{1}$ and $A_{2}$. 
Remark 3. The map $d_{s}$ defined in Proposition 4.4 can be naturally extended to the case where $n=2$. However, in this case $d_{s}$ no longer satisfies condition (i) and hence is not a 2-distance. Indeed, for any $A, B, Z \in \mathbb{R}^{2}$, with $A, B$ distinct, we have

$$
d_{s}(A, B) \leq 2\left(d_{s}(A, Z)+d_{s}(Z, B)\right),
$$

or equivalently,

$$
d(A, B)^{2} \leq 2 d(A, Z)^{2}+2 d(Z, B)^{2},
$$

where the constant 2 is optimal (take $A$ and $B$ distinct and $Z=(A+B) / 2$ ). To see that this inequality holds, set $A=(0,0), B=(b, 0)$, and $Z=(x, y)$. Then, the inequality becomes

$$
b^{2} \leq 2\left(x^{2}+y^{2}\right)+2(x-b)^{2}+2 y^{2},
$$

which always holds because it is algebraically equivalent to

$$
(2 x-b)^{2}+4 y^{2} \geq 0 .
$$

Remark 4. In an attempt to generalize the previous two propositions to $\mathbb{R}^{k}(k \geq 2)$, we may consider the following open questions:

(a) Prove (or disprove) that Proposition 4.3 still holds in $\mathbb{R}^{k}$.

(b) Prove (or disprove) that, for any $n \geq 3$, the map $d_{v}:\left(\mathbb{R}^{k}\right)^{n} \rightarrow \mathbb{R}_{+}$that associates with any $\left(x_{1}, \ldots, x_{n}\right) \in\left(\mathbb{R}^{k}\right)^{n}$ the $k$-dimensional volume bounded by $S\left(x_{1}, \ldots, x_{n}\right)$ is an $n$-distance for which we have $K_{n}^{*}=\left(n-2+2^{1-k}\right)^{-1}$.

Note that the problem in (b) above is motivated by the fact that the corresponding simplex inequality with $K_{n}=\left(n-2+2^{1-k}\right)^{-1}$ holds when $x_{1}$ and $x_{2}$ are distinct and $x_{3}=\cdots=$ $x_{n}=z$ is the midpoint of $x_{1}$ and $x_{2}$.

We now show that counting the number of different directions defined by pairs of distinct elements among $n$ points in the plane defines an $n$-distance.

For any distinct $x, y \in \mathbb{R}^{2}$, we denote by $\overline{x y}$ the direction $\pm(x-y) /\|x-y\|$. Here we assume that $\overline{x y}$ and $\overline{y x}$ represent the same direction.

Proposition 4.5 (Number of directions in $\mathbb{R}^{2}$ ). For any $n \geq 3$, the map $d_{n}:\left(\mathbb{R}^{2}\right)^{n} \rightarrow \mathbb{R}_{+}$ that associates with any $\left(x_{1}, \ldots, x_{n}\right) \in\left(\mathbb{R}^{2}\right)^{n}$ the cardinality $|\Delta|$ of the set

$$
\Delta=\left\{\overline{x_{i} x_{j}} \mid i, j \in[n] \text { and } x_{i} \neq x_{j}\right\}
$$

is an $n$-distance for which we have $\frac{1}{n-2+\frac{2}{n}} \leq K_{n}^{*}<\frac{1}{n-2}$.

Proof. Let $x_{1}, \ldots, x_{n}, z \in \mathbb{R}^{2}$. For any $i \in[n]$, let

$$
\Delta_{i}=\left\{\overline{x_{j} x_{k}} \mid j, k \in[n] \backslash\{i\} \text { and } x_{j} \neq x_{k}\right\} .
$$

On the one hand, we clearly have $\left|\Delta_{i}\right| \leq d_{n}\left(x_{1}, \ldots, x_{n}\right)_{i}^{z}$ for every $i \in[n]$. On the other hand, it is easy to see that each direction in $\Delta$ is counted at least $(n-2)$ times in the sum $\sum_{i=1}^{n}\left|\Delta_{i}\right|$. From these observations it follows that

$$
(n-2) d_{n}\left(x_{1}, \ldots, x_{n}\right)=(n-2)|\Delta| \leq \sum_{i=1}^{n}\left|\Delta_{i}\right| \leq \sum_{i=1}^{n} d_{n}\left(x_{1}, \ldots, x_{n}\right)_{i}^{z},
$$

which proves that $K_{n}^{*} \leq \frac{1}{n-2}$.

We now show by contradiction that the latter inequality is strict. Assume that there exist $x_{1}, \ldots, x_{n}, z \in \mathbb{R}^{2}$ such that

$$
(n-2) d_{n}\left(x_{1}, \ldots, x_{n}\right)=\sum_{i=1}^{n} d_{n}\left(x_{1}, \ldots, x_{n}\right)_{i}^{z} .
$$


It follows that for these points we can replace both inequalities in 25) with equalities. The first equality then means that each direction in $\Delta$ is counted exactly $(n-2)$ times in the sum $\sum_{i=1}^{n}\left|\Delta_{i}\right|$. It is easy to see that this condition also means that no three of the points $x_{1}, \ldots, x_{n}$ are collinear. Let us now consider the second inequality. Since $\left|\Delta_{i}\right| \leq d_{n}\left(x_{1}, \ldots, x_{n}\right)_{i}^{z}$ for every $i \in[n]$, we must have $\left|\Delta_{i}\right|=d_{n}\left(x_{1}, \ldots, x_{n}\right)_{i}^{z}$ for every $i \in[n]$. Suppose first that $n \geq 4$. It follows from the latter condition that both sets $\left\{x_{2}, \ldots, x_{n}\right\}$ and $\left\{z, x_{2}, \ldots, x_{n}\right\}$ generate the same number of directions. Since no three of the points $x_{2}, \ldots, x_{n}$ are collinear, we should have $z=x_{\ell}$ for some $\ell \in\{2, \ldots, n\}$. But then we have $\left|\Delta_{\ell}\right|<d_{n}\left(x_{1}, \ldots, x_{n}\right)_{\ell}^{z}$, a contradiction. A similar contradiction can be easily reached when $n=3$.

Let us now establish the lower bound for $K_{n}^{*}$. Let $x_{1}, \ldots, x_{n}$ be pairwise distinct and placed clockwise on the unit circle. Let also $z=x_{1}$. Then we have

$$
d_{n}\left(x_{1}, \ldots, x_{n}\right)=\left(\begin{array}{l}
n \\
2
\end{array}\right) \text { and } d_{n}\left(x_{1}, \ldots, x_{n}\right)_{i}^{z}= \begin{cases}\left(\begin{array}{c}
n \\
2
\end{array}\right) & \text { if } i=1, \\
\left(\begin{array}{c}
n-1 \\
2
\end{array}\right) & \text { if } i \neq 1,\end{cases}
$$

and hence

$$
\begin{aligned}
\sum_{i=1}^{n} d_{n}\left(x_{1}, \ldots, x_{n}\right)_{i}^{z} & =\left(\begin{array}{l}
n \\
2
\end{array}\right)+(n-1)\left(\begin{array}{c}
n-1 \\
2
\end{array}\right)=\left(n-2+\frac{2}{n}\right)\left(\begin{array}{l}
n \\
2
\end{array}\right) \\
& =\left(n-2+\frac{2}{n}\right) d_{n}\left(x_{1}, \ldots, x_{n}\right),
\end{aligned}
$$

which completes the proof.

Remark 5. An $n$-distance $d:\left(\mathbb{R}^{k}\right)^{n} \rightarrow \mathbb{R}_{+}$is said to be homogeneous of degree $q \geq 0$ if, for any $t>0$, we have

$$
d\left(t x_{1}, \ldots, t x_{n}\right)=t^{q} d\left(x_{1}, \ldots, x_{n}\right), \quad x_{1}, \ldots, x_{n} \in \mathbb{R}^{k} .
$$

This means that under any dilation $x \mapsto t x$, the $n$-distance $d$ is magnified by the factor $t^{q}$. Since a distance on $\mathbb{R}^{k}$ usually represents a linear dimension, we could expect any $n$-distance on $\mathbb{R}^{k}$ to be homogeneous of degree 1 . This is for instance the case for the $n$-distance defined in Proposition 4.3. Surprisingly enough, the $n$-distances defined in Examples 2.1, 2.2 and Proposition 4.5 are homogeneous of degree 0, that is, invariant under any dilation. Also, the $n$-distance defined in Proposition 4.4 is homogeneous of degree 2 .

\section{A GeneralizATION OF THE CONCEPT OF $n$-DistANCE}

The concept of $n$-distance as defined in Definition 1.1 can naturally be generalized by relaxing condition (i) as follows.

Definition 5.1. Let $g: \mathbb{R}_{+}^{n} \rightarrow \mathbb{R}_{+}$be a symmetric function, i.e., invariant under any permutation of its arguments. We say that a function $d: X^{n} \rightarrow \mathbb{R}_{+}$is a $g$-distance if it satisfies conditions (ii), (iii), and

$$
d\left(x_{1}, \ldots, x_{n}\right) \leqslant g\left(d\left(x_{1}, \ldots, x_{n}\right)_{1}^{z}, \ldots, d\left(x_{1}, \ldots, x_{n}\right)_{n}^{z}\right)
$$

for all $x_{1}, \ldots, x_{n}, z \in X$.

In view of Proposition 2.8 it is natural to require $d+d^{\prime}, \lambda d$, and $\frac{d}{1+d}$ to be $g$-distances whenever so are $d$ and $d^{\prime}$. The following proposition provides sufficient conditions on $g$ for these properties to hold. Recall that a function $g: \mathbb{R}_{+}^{n} \rightarrow \mathbb{R}$ is positively homogeneous if $g(\lambda \mathbf{r})=\lambda g(\mathbf{r})$ for all $\mathbf{r} \in \mathbb{R}_{+}^{n}$ and all $\lambda>0$. It is said to be superadditive if $g(\mathbf{r}+\mathbf{s}) \geq$ 
$g(\mathbf{r})+g(\mathbf{s})$ for every $\mathbf{r}, \mathbf{s} \in \mathbb{R}_{+}^{n}$. Also, it is additive if $g(\mathbf{r}+\mathbf{s})=g(\mathbf{r})+g(\mathbf{s})$ for every $\mathbf{r}, \mathbf{s} \in \mathbb{R}_{+}^{n}$.

Proposition 5.2. Let $g: \mathbb{R}_{+}^{n} \rightarrow \mathbb{R}_{+}$be a symmetric function, and let $d, d^{\prime}: X^{n} \rightarrow \mathbb{R}_{+}$be $g$-distances. The following assertions hold.

(a) If $g$ is positively homogeneous, then $\lambda d$ is a g-distance for every $\lambda>0$.

(b) If $g$ is superadditive, then $d+d^{\prime}$ is a $g$-distance.

(c) If $g$ is both positively homogeneous and superadditive, then it is concave.

(d) The function $g$ is additive if and only if there exists $\lambda \geq 0$ such that

$$
g(\mathbf{r})=\lambda \sum_{i=1}^{n} r_{i}, \quad \mathbf{r}=\left(r_{1}, \ldots, r_{n}\right) \in \mathbb{R}_{+}^{n} .
$$

(e) If $g$ satisifies (26) for some $\lambda \geq 1$, then $\frac{d}{1+d}$ is a g-distance.

Proof. (a) and (b) follow from the definitions.

(c) For any $\lambda \in[0,1]$, we have

$$
g(\lambda \mathbf{r}+(1-\lambda) \mathbf{s}) \leq g(\lambda \mathbf{r})+g((1-\lambda) \mathbf{s})=\lambda g(\mathbf{r})+(1-\lambda) g(\mathbf{s}),
$$

where the inequality follows from superadditivity and the equality from positive homogeneity.

(d) The sufficiency is trivial. To see that the necessity holds, note that $g$ is additive and bounded from below (since it ranges in $\mathbb{R}_{+}$) and hence it is continuous and there exist $\lambda_{1}, \ldots, \lambda_{n} \in \mathbb{R}$ such that $g(\mathbf{r})=\sum_{i=1}^{n} \lambda_{i} r_{i}$; see [1, Cor. 2, p. 35]. The result then follows from the symmetry of $g$.

(e) Let $x_{1}, \ldots, x_{n}, z \in X$ and set $d=d\left(x_{1}, \ldots, x_{n}\right)$ and $d_{i}=d\left(x_{1}, \ldots, x_{n}\right)_{i}^{z}$ for every $i \in[n]$. Since $\lambda \geq 1$, we have $\lambda r /(1+\lambda r) \leq \lambda r /(1+r)$ for every $r \geq 0$. It then follows that

$$
\frac{1}{1+d} \leq \sum_{i=1}^{n} \frac{\lambda d_{i}}{1+\lambda d_{i}} \leq \sum_{i=1}^{n} \frac{\lambda d_{i}}{1+d_{i}}
$$

where the first inequality follows from Lemma2.7 and the fact that $d$ is a $g$-distance.

\section{CONCLUSION AND FURTHER RESEARCH}

In this paper we have introduced and discussed the concept of $n$-distance as a natural generalization of the concept of distance to functions of $n \geq 2$ variables. There are two key features in this generalization: one is an $n$-ary version of the identity of indiscernibles, and the other is the simplex inequality, which is a natural generalization of the triangle inequality. We have observed that any $n$-distance $d$ has an associated best constant $\left.\left.K_{n}^{*} \in\right] 0,1\right]$ satisfying inequality (11). Also, we have provided many natural examples of $n$-distances, and have shown that searching for their associated best constant may be mathematically challenging and may sometimes require subtle arguments. The examples we have discussed might suggest that we have $K_{n}^{*}<1$ for any $n$-distance. The following example, which was communicated to us by Roberto Ghiselli Ricci [25], shows that this is not the case.

Example 6.1. Let $n \geq 3$ and $a \in \mathbb{R}$. Let also $\mathcal{A}(a, n)$ be the set of $n$-tuples whose components are consecutive elements of arithmetic progressions with common difference $a$. Consider the map $d_{n}: \mathbb{R}^{n} \rightarrow \mathbb{R}_{+}$defined as

$$
d_{n}\left(x_{1}, \ldots, x_{n}\right)= \begin{cases}0 & \text { if } x_{1}=\cdots=x_{n}, \\ 1 & \text { if }\left(x_{1}, \ldots, x_{n}\right) \in \mathcal{A}(a, n) \text { for some } a \neq 0, \\ \frac{1}{n} & \text { otherwise. }\end{cases}
$$


We prove that $d_{n}$ is an $n$-distance for which we have $K_{n}^{*}=1$. Conditions (ii) and (iii) are easily verified. To see that condition (i) holds, consider $x_{1}, \ldots, x_{n}, z \in \mathbb{R}$. First assume that $d_{n}\left(x_{1}, \ldots, x_{n}\right)=\frac{1}{n}$. There is at most one $i \in[n]$ such that $d_{n}\left(x_{1}, \ldots, x_{n}\right)_{i}^{z}=0$. Thus, we obtain

$$
\sum_{i=1}^{n} d_{n}\left(x_{1}, \ldots, x_{n}\right)_{i}^{z} \geq \frac{n-1}{n} \geq d_{n}\left(x_{1}, \ldots, x_{n}\right) .
$$

Assume now that $d\left(x_{1}, \ldots, x_{n}\right)=1$. It follows that $d_{n}\left(x_{1}, \ldots, x_{n}\right)_{i}^{z} \geq \frac{1}{n}$ for all $i \in[n]$, which shows that the simplex inequality holds in that case as well. To prove that $K_{n}^{*}=1$, just consider $x_{1}=1, x_{2}=2, \ldots, x_{n}=n$, and $z=-1$.

We also observe that certain $n$-distances cannot be constructed from the concept of multidistance as defined by Martín and Mayor [19] (see Remark 1). Instances of such $n$-distances are given, e.g., in Propositions 4.4 and 4.5

We conclude this paper by proposing a few topics for further research.

(a) Improve the bounds for the best constant associated with the Fermat $n$-distance (at least in some given proper metric spaces).

(b) Consider and solve the problems stated in Remark 4

(c) Investigate properties of topological spaces based on $n$-metric spaces. On this issue we observe that in [24] the authors introduced a stronger version of 3-metric space called $G$-metric space (see also [16]). It is shown that there is a natural metric space associated with any $G$-metric space. Finding an appropriate generalization of the notion of $G$-metric space as a stronger version of $n$-metric space and investigating its topological properties seems to be an interesting topic of research.

\section{ACKNOWLEDGMENTS}

This research is supported by the internal research project R-AGR-0500 of the University of Luxembourg.

\section{REFERENCES}

[1] J. Aczél and J. Dhombres. Functional equations in several variables. Encyclopedia of Mathematics and its Applications, vol. 31. Cambridge University Press, Cambridge, 2008.

[2] I. Aguiló, J. Martín, G. Mayor, J. Suñer, and O. Valero. Smallest enclosing ball multidistance. Communications in Information and Systems 12(3):185-194, 2012.

[3] V. Boltyanski, H. Martini, and V. Soltan. Geometric Methods and Optimization Problems. Kluwer Academic Publishers, Dordrecht, 1999.

[4] G. Chrystal. On the problem to construct the minimum circle enclosing $n$ given points in a plane. Proc. of the Edinburgh Math. Soc., Vol. 3, pp. 30-33, 1884.

[5] M. M. Deza and E. Deza. Encyclopedia of distances, third edition. Springer, 2014.

[6] M. M. Deza and M. Dutour. Cones of metrics, hemi-metrics and super-metrics. Ann. of European Academy of Sci. 1:146-162, 2003.

[7] M. M. Deza and I. G. Rosenberg. n-semimetrics. European Journal of Combinatorics, 21:97-806, 2000.

[8] B. C. Dhage. Generalised metric spaces and mappings with fixed point. Bulletin of the Calcutta Mathematical Society 84:329-336, 1992.

[9] B. C. Dhage. On generalized metric spaces and topological structure. II. Pure and Applied Mathematika Sciences 40(1-2):37-41, 1994.

[10] B. C. Dhage. On the continuity of mappings in D-metric spaces. Bulletin of the Calcutta Mathematical Society 86:503-508, 1994.

[11] B. C. Dhage. Generalized metric spaces and topological structure. I. Analele Ştiinţifice ale Universităţii "Al. I. Cuza” din Iaşi 46(1):31-38, 2000.

[12] Z. Drezner, K. Klamroth, A. Schöbel, and G. O. Wesolowsky. The Weber Problem. In: Facility Location, Application and Theory. Z. Drezner, and H. W. Hamacher, Editors. Springer-Verlag Berlin, pp 1-24, 2002. 
[13] M. Fréchet. Sur quelques points de calcul fonctionnel. Rendiconti del Circolo Matematico di Palermo 22:172, 1906.

[14] F. Hausdorff. Grundzüge der Mengenlehre. Veit and Company, Leipzig, 1914.

[15] T. Jahn, Y. S. Kupitz, H. Martini, C. Richter. Minsum location extended to gauges and to convex sets. J. Optim. Theory Appl. 166(3):711-746, 2015.

[16] M. A. Khamsi. Generalized metric spaces: A survey. Journal of Fixed Point Theory and Applications, 17:455-475, 2015.

[17] G. Kiss, J.-L. Marichal, and B. Teheux. An extension of the concept of distance as functions of several variables. Proc. 36th Linz Seminar on Fuzzy Set Theory (LINZ 2016), Linz, Austria, Feb. 2-6, pp. 53-56, 2016.

[18] J. Martín and G. Mayor. Some properties of multiargument distances and Fermat multidistance. In Information Processing and Management of Uncertainty in Knowledge-Based Systems. Theory and Methods. 13th Int. Conf., IPMU 2010, Dortmund, Germany, June 28-July 2, 2010. Proceedings, Part I. Volume 80 of the series Communications in Computer and Information Science. Springer, pp. 703-711, 2010.

[19] J. Martín and G. Mayor. Multi-argument distances. Fuzzy Sets and Systems 167:92-100, 2011.

[20] J. Martín, G. Mayor, and O. Valero. Functionally expressible multidistances. In: EUSFLAT-LFA Conf. July 2011, Aix-les-Bains, France. Series: Advances in Intelligent Systems Research. Atlantis Press, pp. 41-46, 2011.

[21] N. Megiddo. Linear-time algorithms for linear programming in $\mathbb{R}^{3}$ and related problems. SIAM Journal on Computing 12(4), 759-776, 1983.

[22] N. Megiddo. Linear programming in linear time when the dimension is fixed. Journal of the ACM 31(1):114127, 1984.

[23] Z. Mustafa and B. Sim. Some remarks concerning D-metric spaces. In: Proceedings of the International Conference on Fixed Point Theory and Applications (Valencia, Spain, 2003), Yokohama Publ., Yokohama, 2001, 189-198.

[24] Z. Mustafa and B. Sims. A new approach to generalized metric spaces. Journal of Nonlinear and Convex Analysis, 7(2): 289-297, 2006.

[25] R. G. Ricci. University of Ferrara, Italy. Personal communication, 2016.

[26] E. Welzl. Smallest enclosing disks (balls and ellipsoids). In: New Results and New Trends in Computer Science. Lecture Notes in Computer Science vol. 555, Springer, pp. 359-370, 1991.

Mathematics Research Unit, University of Luxembourg, Maison du Nombre, 6, avenue DE LA FONTE, L-4364 ESCH-SUR-ALZETTE, LUXEMBOURG

E-mail address: gergely.kiss [at] uni.lu

Mathematics Research Unit, University of Luxembourg, Maison du Nombre, 6, avenue DE LA FONTE, L-4364 ESCH-SUR-ALZETTE, LUXEMBOURG

E-mail address: jean-luc.marichal [at] uni.lu

Mathematics Research Unit, University of Luxembourg, Maison du Nombre, 6, avenue DE LA FonTE, L-4364 ESCH-SUR-ALZETTE, LUXEMBOURG

E-mail address: bruno.teheux [at ] uni. lu 